\title{
COMPUTERIZED MODELING OF KINEMATICS AND KINETO-STATICS OF SUCKER-ROD PUMP POWER UNITS
}

\author{
Sayat Ibraev ${ }^{1}$, Aiman Nurmaganbetova ${ }^{1}$, Nurbibi Imanbaeva ${ }^{1,2}$, Algazy Zhauyt $^{2}$ \\ ${ }^{1}$ Institute of Mechanics and Mechanical Engineering named after Academician U. A. Joldasbekov of \\ the Committee for Science of the Ministry of Education and Science of the Republic of Kazakhstan, \\ ${ }^{2}$ Kazakh National Research Technical University named after K. I. Satpaev, Kazakhstan \\ ibraev@mail.ru, aiman_n@mail.ru, imanbaevan@mail.ru, ali84jauit@mail.ru
}

\begin{abstract}
The paper outlines the methodology of kinematic and kineto-static analysis of the converting mechanism of sucker-rod pump power units (SPPU). The key point of stated technique is that the research is based on an analytic approach followed by obtaining of accurate results. Numerical results of the kinematic and kineto-static analysis are given. Computerzed modeling is made based on the research performed, which will allow to investigate the modes of optimal dynamic balancing of the converting mechanism of SPPU in the future.
\end{abstract}

Key words: sucker-rod, kinematic, absolute coordinates, walking beam, equalizer beam.

\section{Introduction}

Development of mechanical engineering in the oil and gas industry is one of the priorities [1]. The problem of produced oil cost reduction is particularly acute. The most common of the existing mechanized methods of oil production is the sucker-rod deep-well with balanced individual pump power units of mechanical action [2]. Power units are designed to actuate the deep-well pump plunger into a reciprocating motion using rods. Balanced pumping jacks have a simple, well-studied diagram and cost effective construction design maintainability and reliability compared with other power units. Approximate formulas were used for modeling the construction of the existing standard sizes of pumping jacks at the design development phase [3-4]. The lack of a reliable and effective tool for their modeling and optimal design are one of the main reasons that the existing units are poorly balanced [5]. Analysis of two-drive group of Assur was used to analyze the positions, velocities and accelerations of pivot joints, force application points (centers of links and element masses) of the converting mechanism of the power unit (SPPU), which is based on an analytic approach followed by obtaining of accurate results [6-11].

\section{Materials and Methods}

Kinematics diagram of the converting mechanism of SPPU power unit with a rocker double-arm (see Fig. 1) is a crank-and-rocker articulation linkage mechanism with a crank OA, connecting rod $\mathrm{AB}$ and rocker arm $C B$. The rocker arm has an arched horse-head for converting the rocking motion of the rocker arm part into the reciprocating motion of rods to be connected to the horse-head using flexible (wire-line) link $D E$. Absolute coordinates of the pivot joints $C$ and $A_{i}, i=1, \ldots, N$ are considered as known at the given crank angles $\varphi_{i}=\left(\varphi_{O A}\right), i=1, \ldots, N$, and the length of the links $A B=l_{b}$ and $B C$, where the last was determined through the specified sizes $k, b \delta, b B$ (see Fig. 1):

$$
B C=\left(k^{2}+(b \delta-b B)^{2}\right)^{1 / 2} .
$$

When leaving the sub-program, we have angular positions of the rocker arm and walking beam, absolute coordinates of the pivot joint $B$. Crank angles $\varphi_{\text {bot }}$ and $\varphi_{t o p}$, corresponding to the end positions of the rod suspension point, were determined using the same sub-program. Below the determination of absolute coordinates of all points of interest to us is given: pivot joints, points of forces applications (centers of links and element masses). Coordinate of the pivot joint $A$ of the crank arm:

$$
\begin{aligned}
& X_{A}=X_{O}+O A \cdot \cos \varphi_{1} \\
& Y_{A}=Y_{O}+O A \cdot \sin \varphi_{1}
\end{aligned}
$$

Coordinates $X_{B}, Y_{B}$ of the pivot joint $B$ :

$$
X_{B}=X_{A}+A B \cdot \cos \varphi_{2}, \quad Y_{B}=Y_{A}+A B \cdot \sin \varphi_{2} .
$$

Angle $\varphi_{2}$ is determined by formula: 


$$
\begin{gathered}
\varphi_{2}=\operatorname{arctg}\left[\frac{X_{A}-X_{C}}{Y_{A}-Y_{C}}\right]+\arccos \left[\frac{l_{A B}^{2}+S_{i}^{2}-l_{A B}^{2}}{2 l_{A B} \cdot S_{i}}\right], \\
S_{i}=\sqrt{\left(X_{A}-X_{C}\right)^{2}+\left(Y_{A}-Y_{C}\right)^{2}} .
\end{gathered}
$$

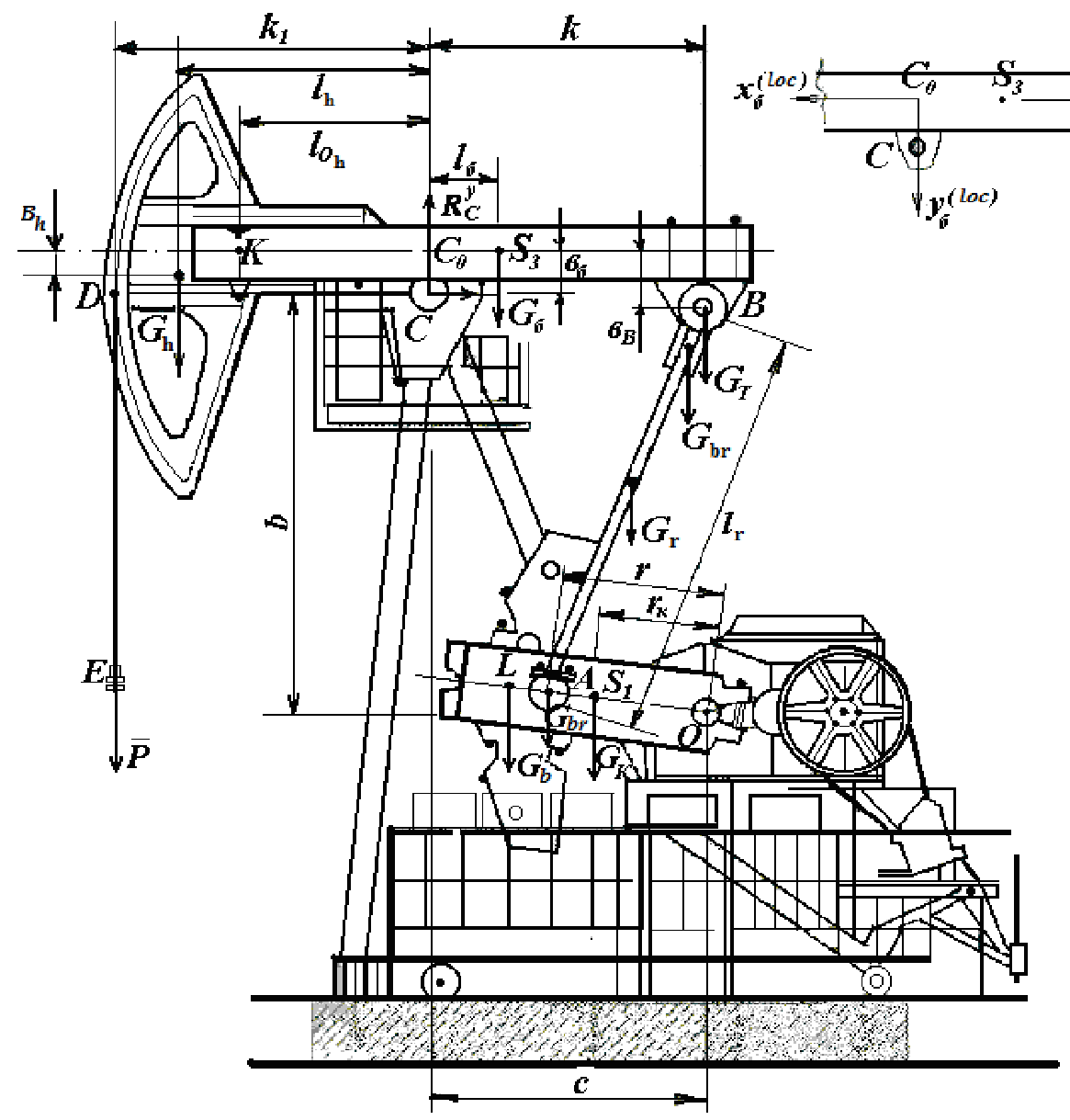

Fig. 1. Converting mechanism of sucker-rod pump power unit

Coordinates of the center of masses of the equalizer beam:

$$
\begin{gathered}
\left\{\begin{array}{l}
X_{\text {Trav }}=X_{B}+\frac{B T\left(X_{A}-X_{B}\right)}{A B} \\
Y_{\text {Trav }}=Y_{B}+\frac{B T\left(Y_{A}-Y_{B}\right)}{A B},
\end{array}\right. \\
X_{C_{0}}=X_{C}+y_{C_{\text {loc }}} \sin \varphi_{3}, \quad Y_{C_{0}}=Y_{C}-y_{C_{\text {loc }}} \cos \varphi_{3},
\end{gathered}
$$

where $y_{C_{l o c}}$ - local coordinate:

$$
\begin{gathered}
\varphi_{3}=\operatorname{arctg}\left[\frac{Y_{B}-Y_{C}}{X_{B}-X_{C}}\right], \\
X_{B}=X_{A}+A B \cos \varphi_{2}, \quad Y_{B}=Y_{A}+A B \sin \varphi_{2} .
\end{gathered}
$$

Coordinates of the connection point of the walking beam and swinging horse-head: 


$$
\left\{\begin{array}{l}
X_{D}=X_{C_{0}}+x_{D_{l o c}} \cos \varphi_{3}-y_{D_{l o c}} \sin \varphi_{3} \\
Y_{D}=Y_{C_{0}}+x_{D_{l o c}} \sin \varphi_{3}+y_{D_{l o c}} \cos \varphi_{3}
\end{array} .\right.
$$

Coordinates of the center of masses of the arched folding horse-head:

$$
\left\{\begin{array}{l}
X_{\text {head }}=X_{C_{0}}+x_{H_{l o c}} \cos \varphi_{3}-y_{H_{l o c}} \sin \varphi_{3} \\
Y_{\text {head }}=Y_{C_{0}}+x_{H_{\text {loc }}} \sin \varphi_{3}+y_{H_{\text {loc }}} \cos \varphi_{3}
\end{array} .\right.
$$
beam):

Coordinates of the center of masses of the walking beam (without horse-head and equalizer

$$
\left\{\begin{array}{l}
X_{S_{3}}=X_{C_{0}}+x_{3_{l o c}} \cos \varphi_{3}-y_{3_{l o c}} \sin \varphi_{3} \\
Y_{S_{3}}=Y_{C_{0}}+x_{3_{l o c}} \sin \varphi_{3}+y_{3_{l o c}} \cos \varphi_{3}
\end{array} .\right.
$$

Coordinates of the rod suspension point:

$$
X_{E}=X_{E_{0}}, \quad Y_{E}=Y_{E_{0}}-C D \cdot \varphi_{3} .
$$

It represents graphical copies of mean and critical configurations of the converting mechanism of the SPPU power unit. After differentiation of Eq. (7) with respect to generalized coordinate $\varphi_{1}$, we obtain:

$$
\varphi_{3}^{\prime}=\frac{l_{1}\left(-X_{2} \sin \varphi_{1}+Y_{2} \cos \varphi_{1}\right)}{l_{3}\left(-X_{2} \sin \varphi_{3}+Y_{2} \cos \varphi_{3}\right)} .
$$

Projections of velocity of the pivot joint $A$ on the coordinate axes:

$$
V_{A}^{X}=-\omega_{1}\left(X_{A}-X_{O}\right), \quad V_{A}^{Y}=\omega_{1}\left(Y_{A}-Y_{O}\right) .
$$

Projections of velocity of the center of masses of the crank arm:

$$
V_{S 1}^{X}=-\omega_{1}\left(X_{S 1}-X_{O}\right), \quad V_{S 1}^{Y}=\omega_{1}\left(Y_{S 1}-Y_{O}\right) .
$$

Projections of velocity of the counter balance location point:

$$
V_{S t}^{X}=-\omega_{1}\left(X_{S t}-X_{O}\right), \quad V_{S t}^{Y}=\omega_{1}\left(Y_{S t}-Y_{O}\right) .
$$

Velocity of the pivot joint $B$ in projections:

$$
V_{B}^{X}=-\omega_{3}\left(X_{B}-X_{C}\right), \quad V_{B}^{Y}=\omega_{3}\left(Y_{B}-Y_{C}\right) .
$$

Velocity of the center of masses of the equalizer beam:

$$
V_{\text {Trav }}^{X}=V_{B}^{X}+l_{C T} \frac{\left(V_{A}^{X}-V_{B}^{X}\right)}{s_{2}}, V_{\text {Trav }}^{Y}=V_{B}^{Y}+l_{C T} \frac{\left(V_{A}^{Y}-V_{B}^{Y}\right)}{s_{2}} .
$$

Velocity of the center of masses of the connecting rod:

$$
V_{S 2}^{X}=\frac{V_{B}^{X}+V_{A B}^{X}}{2}, \quad V_{S 2}^{Y}=\frac{V_{B}^{Y}+V_{A B}^{Y}}{2} .
$$

Velocity of the center of masses of the horse-head and arched horse-head:

$$
\begin{array}{cc}
V_{S 3}^{X}=-\omega_{3}\left(X_{S 3}-X_{C}\right), & V_{S 3}^{Y}=\omega_{3}\left(Y_{S 3}-Y_{C}\right), \\
V_{G}^{X}=-\omega_{3}\left(X_{G}-X_{C}\right), & V_{G}^{Y}=\omega_{3}\left(Y_{G}-Y_{C}\right) .
\end{array}
$$

Velocities of the rod suspension point:

$$
V_{K}^{X}=-\omega_{3}\left(X_{K}-X_{C}\right), \quad V_{K}^{Y}=\omega_{3}\left(Y_{K}-Y_{C}\right),
$$




$$
V_{E}^{X}=0, \quad V_{E}^{Y}=\omega_{3}\left(Y_{E}-Y_{C}\right)
$$

\section{Kineto-statics of the converting mechanism of SPPU power unit}

Loads in the well casing-head stuffing box and gravity forces of the links and weights have an impact on the mechanism (see Fig. 1). In the mechanism under consideration (kinematic diagram with the walking beam representing the lever of the second kind) there is an impact of the load in the well casing-head stuffing box and gravity forces of the links and the following weights.

$G_{c a}$ - weight of the crank arm (shall be doubled); $G_{21}=2 \cdot G_{b r}$, where $G_{c a}$ - weight of the bottom connecting rod end; $G_{22}=2 \cdot G_{r}$, where $G_{r}$ - weight of the connecting rod; $G_{21}=2 \cdot G_{b r}$ - weight of the equalizer beam; $G_{31}=G_{T}$ - weight of the equalizer beam support on the walking beam; $G_{32}=G_{b a l}$ weight of the walking beam; $G_{33}=G_{h}$ - weight of the horse-head; $G_{b}$ - buoyant weight; $G_{c o u}$ - weight of the counter balance.

Link 2 is effected by forces:

$$
\vec{F}_{21}=\vec{G}_{21}-m_{21} \vec{a}_{A}, \vec{F}_{22}=\vec{G}_{22}-m_{22} \vec{a}_{S 2}, \vec{F}_{23}=\vec{G}_{23}-m_{23} \vec{a}_{T 2} \text { in points } A, S_{2}, T_{2} .
$$

Link 3 is effected by forces:

$$
\vec{F}_{31}=\vec{G}_{31}-m_{31} \vec{a}_{T 3}, \vec{F}_{32}=\vec{G}_{32}-m_{32} \vec{a}_{S 3}, \vec{F}_{33}=\vec{G}_{33}-m_{33} \vec{a}_{H} \text {, in points } B, S_{3}, H \text {. }
$$

$A$ in point $E$ of string suspension - the load on down stroke:

$$
\vec{F}_{34}=\vec{P}_{b o t}-m_{34} \vec{a}_{E} \text {, on upstroke } \vec{F}_{34}=\vec{P}_{t o p}-m_{34} \vec{a}_{E} .
$$

Let us formulate equilibrium equation to determine the reaction of the two-drive group, which is given for the solution of the following system:

$$
A \cdot \vec{R}=\vec{b},
$$

where

$$
A=\left[\begin{array}{cccccc}
1 & 0 & 1 & 0 & 0 & 0 \\
0 & 1 & 0 & 1 & 0 & 0 \\
0 & 0 & Y_{A}-Y_{C} & X_{A}-X_{C} & 0 & 0 \\
0 & 0 & -1 & 0 & 1 & 0 \\
0 & 0 & 0 & -1 & 0 & 1 \\
0 & 0 & Y_{C}-Y_{B} & X_{C}-X_{B} & 0 & 0
\end{array}\right] ; \quad b=\left[\begin{array}{c}
-F_{1}{ }^{x} \\
-F_{1}{ }^{y} \\
\left(Y_{S 1}-Y_{A}\right) F_{1}{ }^{x}-\left(X_{S 1}-X_{A}\right) F_{1}{ }^{y}-M_{1} \\
-F_{2}{ }^{x} \\
-F_{2}^{y} \\
\left(Y_{S 2}-Y_{B}\right) F_{2}{ }^{x}-\left(X_{S 2}-X_{B}\right) F_{2}{ }^{y}-M_{2}
\end{array}\right], \vec{R}=\left[\begin{array}{c}
R_{1}{ }^{x} \\
R_{1}{ }^{y} \\
R_{21}^{x} \\
R_{21}^{y} \\
R_{2}^{x} \\
R_{2}^{y}
\end{array}\right]
$$

Let us determine the reactions

$$
R_{A}^{x}, R_{A}^{y}, R_{32}^{x}, R_{32}^{y}, R_{C}^{x}, R_{C}^{y}
$$

using known methods as well as normal, tangential component reactions (see Fig. 3, 5). Then, let us calculate the crank arm power. The crank arm is effected by forces

$$
\vec{R}_{21}, \vec{F}_{12}=\vec{G}_{K}-m_{K} \vec{a}_{S 1}, \vec{F}_{13}=\vec{G}_{b}-m_{b} \vec{a}_{L}, \vec{F}_{14}=\vec{G}_{\text {Red }} \text { and in points } A, S_{1}, L \text {. }
$$

Also let us determine $\vec{M}, R_{01}^{x}, R_{01}^{y}$. The results of the kineto-statics research are reflected in Fig. 2-5.

\section{Results and discussion}

A proper kinematic model has been developed within Visual Basic program to provide information on the feasibility of the proposed design solution. Simulation tests have been carried out and the results are discussed for validating the proposed design and characterizing its operation. 
a)

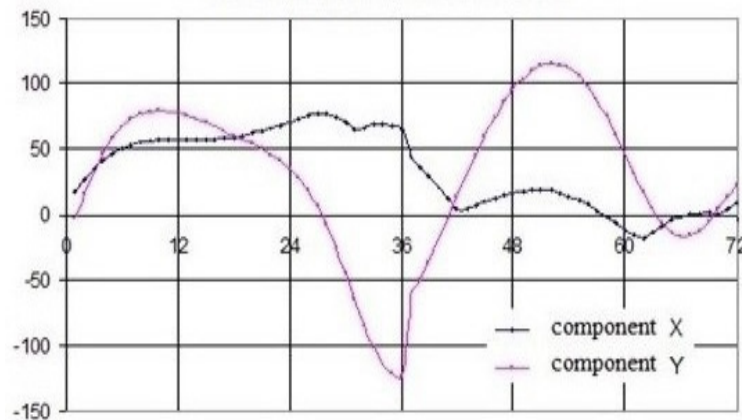

b)

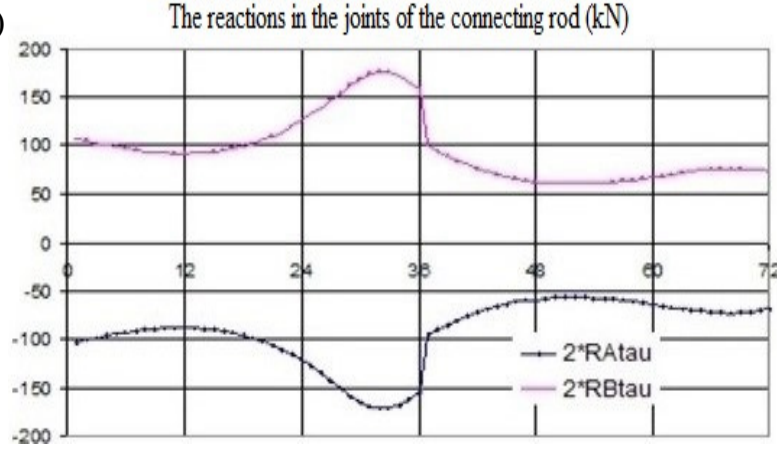

Fig. 2. Behavior of reactions $(\mathbf{k N})$ on output shaft of gear (a) projections on fixed axes and in pivot joints $A$ and $B$ of connecting rod (b) projection along connecting rod at initial data: $n=6.8 \mathrm{vol} \cdot \mathrm{min}^{-1} ; r=1.195 \mathrm{~m} ; P_{B}=80 \mathrm{kN} ; P_{H}=53 \mathrm{kN}$

a)

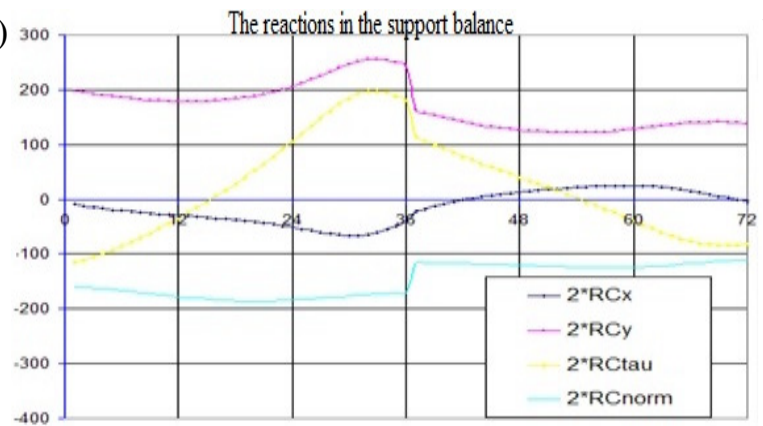

b)

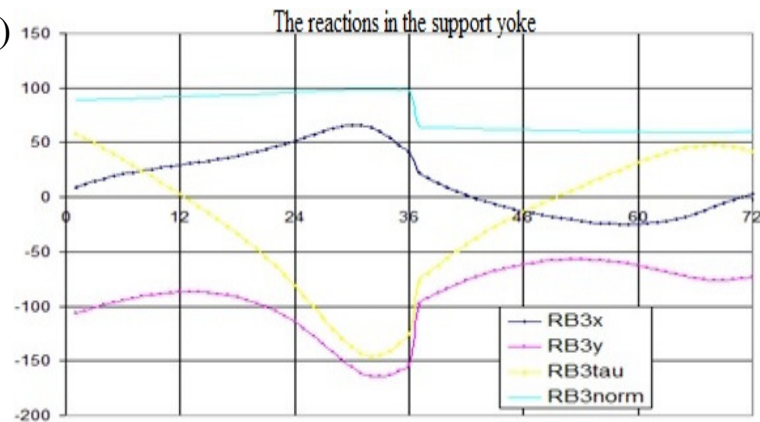

Fig. 3. Behavior of reaction forces $(\mathbf{k N})$ in walking beam support $\mathbf{C}(\mathrm{a})$ and equalizer beam support B (b) at initial data: $n=6.8 \mathrm{vol} \cdot \mathrm{min}^{-1} ; r=1.195 \mathrm{~m} ; P_{B}=80 \mathrm{kN} ; P_{H}=53 \mathrm{kN}$

a)

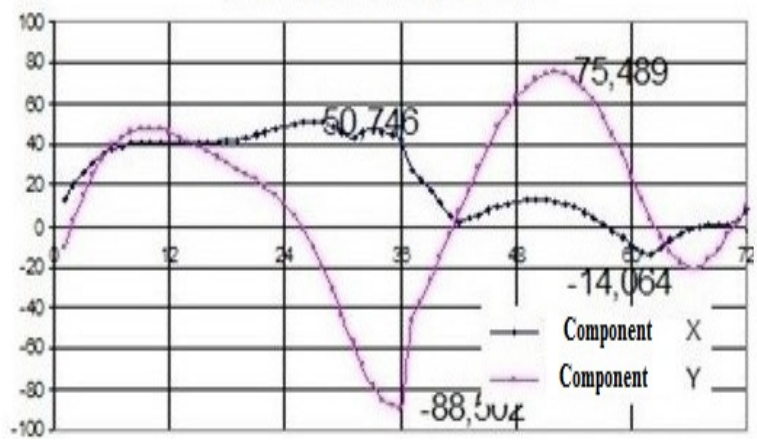

b)

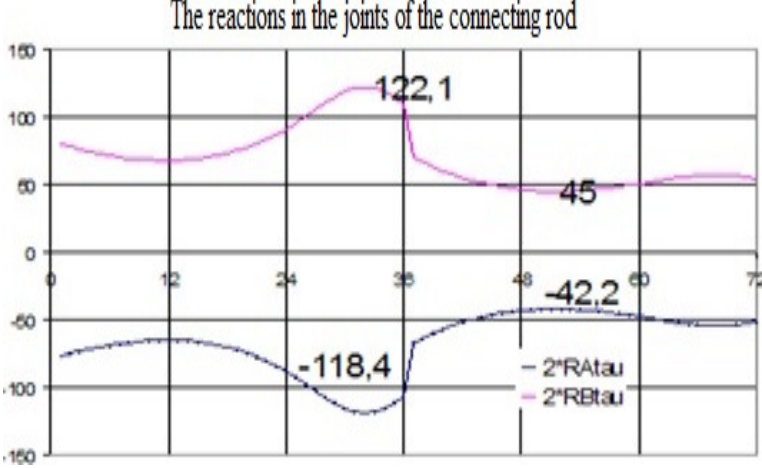

Fig. 4. Behavior of reactions $(\mathbf{k N})$ at initial data (a) - in pivot joint $\mathrm{O}$ (component parts in absolute system of coordinates); (b) - in pivot joints of piston rod (component parts along piston rod axis): $n=6.9 \mathrm{vol} \cdot \mathrm{min}^{-1} ; r=1.000 \mathrm{~m} ; P_{B}=60 \mathrm{kN} ; P_{H}=40 \mathrm{kN}$

a)

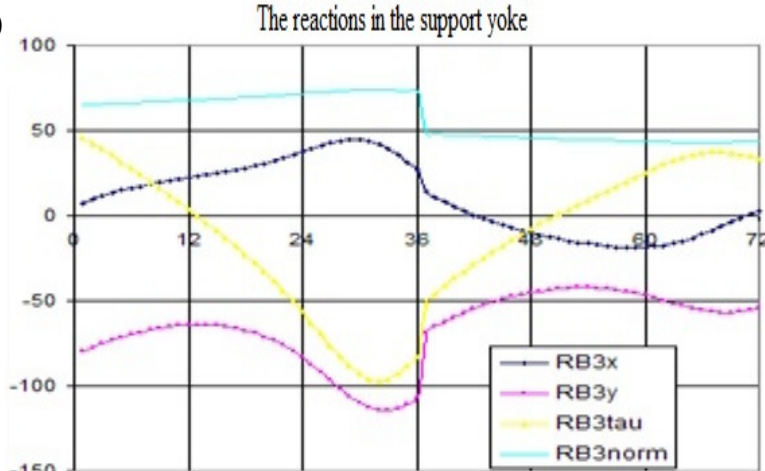

b)

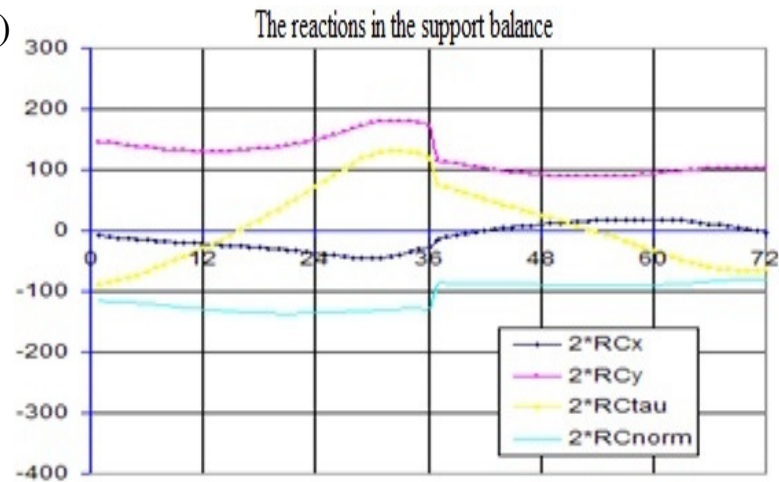

Fig. 5. Behavior of reactions $(\mathrm{kN})$ in pivot joint of equalizer beam $B$ (a) and in support of walking beam C; (b) at initial data: $n=6.9 \mathrm{vol} \cdot \mathrm{min}^{-1} ; r=1.000 \mathrm{~m} ; P_{B}=60 \mathrm{kN} ; P_{H}=40 \mathrm{kN}$ 


\section{Conclusions}

Thus, the results of the research being performed are as follows.

1. Analysis of the converting mechanism positions is made.

2. Graphical copies of mean and critical configurations of the converting mechanism of the SPPU power unit are represented.

3. Values of velocities and accelerations of all pivot joints of the mechanism under consideration are obtained.

4. Values of reactions in pivot joints and supports are analytically determined at various values of input parameters, where the power calculations are made individually for each link.

5. In general, the stated material represents the methodology of computerized modeling of kinematics and kineto-statics of sucker-rod pump power units, which allows to investigate the modes of optimal dynamic balancing of the converting mechanism of the SPPU power unit in the future.

\section{References}

1. Aliveradze K. S. Sucker-rod pump power units, Sub-Soils, 1973, pp. 310-324.

2. Joldasbekov U. A., Baigunchekov Zh. Zh., Ibraev S. M. Kinematics and kinetostatics of multidegree of freedom mechanisms of high classes with given relative motions of moveable links. proc. Tenth world congress on the theory of machines and mechanisms of iftomm, Oulu, Finland, 20-24 June, 1999, pp. 592-596.

3. Joldasbekov S. U., Jamalov N. K., Imanbaeva N. S., Nurmaganbetova A. T. Analysis of the linkage mechanisms positions, Proceedings of the international scientific-practical conference "Problems of modern machines mechanics." Russia, 2003, pp. 318-321.

4. Imanbaeva N. S. Synthesis and kinematic analysis of spatial linkage mechanism of class IV proceedings of the international scientific conference "Problems of applied mathematics." Shymkent, 2006, pp. 316-318.

5. Zaitsev V. V., Balakirev V. A. Technology and engineering of operation of oil and gas wells. Sub-Soil, 1986, pp. 224-236.

6. Joldasbekov S. U., Imanbaeva N. S., Nurmaganbetova A. T. Study of kinematics and kinetostatics of self-aligning converting mechanism of sucker-rod pump power units. Abstracts of the international conference on mechanics "Joldasbekov reading" Almaty, 2011, p0. 123-148.

7. Imanbaeva N. S., Nurmaganbetova A. T., Myrzaliev D. S. The problem of synthesis of drive kinematic circuit of multiple mechanisms with parallel topology for optimal power transmission. IX miedzynarodowej naukowi-praktycznej konferencji «Aktualne problemy nowocznych nauk 2013». Vol. 32, 2013, pp. 46-50.

8. Skanderbek Joldasbekov., Sayat Ibraev., Algazy Zhauyt., Aiman Nurmagambetova., Nurbibi Imanbaeva. Modular synthesis of plane lever six-link mechanism of high class. Middle-East. J. Sci. Res., №21 (12), 2014, pp. 2339-2345.

9. Zhauyt Algazy., Alipov Kuanyshkali., Sakenova Aizhan., Zhankeldi Adilet., Abdirova Raushan., Abilkaiyr Zhastalap. The synthesis of four-bar mechanism. Vibroengineering PROCEDIA, Vol. 10, 2016, pp. 486-491.

10. Zhauyt Algazy., Mamatova Gulnar., Alipov Kuanysh., Sakenova Aizhan., Abdirova Raushan. The kinematic analysis of flat lever mechanisms with application of vector calculation. Vibroengineering PROCEDIA, Vol. 8, 2016, pp. 1-5.

11. Algazy Zhauyt. The substantiating of the dynamic parameters of the shaking conveyor mechanism. Vibroengineering PROCEDIA, Vol. 5, 2015, pp. 15-20. 